\title{
Gastrointestinal and nutritional sequelae of bone marrow transplantation
}

\author{
A Papadopoulou, D R Lloyd, M D Williams, P J Darbyshire, I W Booth
}

\begin{abstract}
The nature of the gastrointestinal injury following bone marrow transplantation and its clinical and nutritional sequelae are poorly defined. Prospective assessments of gastrointestinal function, nutritional status, and wellbeing were therefore carried out in 47 consecutive patients $(28$ males, 19 females; mean age 8.4 years) undergoing bone marrow transplant. 31 diarrhoeal episodes (median duration 9.5 days) occurred in 27 patients at a median of 10 days after transplantation. Ninety one per cent of episodes were associated with protein losing enteropathy. Protein losing enteropathy was more severe in graft-versus-host disease (GVHD) comparing with other causes. It led to a substantial fall in serum albumin and there was a negative correlation between faecal $\alpha_{1}$-antitrypsin concentrations and serum albumin. Transient pancreatic insufficiency developed in 18 patients, and pancreatitis in one. Intestinal permeability was normal in 12 patients who had no diarrhoea during the conditioning treatments. Diarrhoeal patients had a significantly greater decrease in nutritional status and wellbeing than patients without diarrhoea. Gastrointestinal injury following bone marrow transplantation is thus complex. Severe protein losing enteropathy in this context suggests the presence of GVHD.

(Arch Dis Child 1996;75:208-213)
\end{abstract}

Keywords: protein losing enteropathy, pancreatic insufficiency, bone marrow transplantation.

Bone marrow transplantation is now a widely used treatment for several malignant and nonmalignant disorders ${ }^{1}$ and offers a radical cure in affected children. However, the procedure is known to be associated with multiple organ failure, which is usually reversible. Transient intestinal failure following bone marrow transplantation is a common clinical problem because, unlike other forms of organ/tissue transplantation, bone marrow transplants often require the use of total body irradiation (TBI) in addition to immunosuppression. Cells with a rapid turnover, such as haemopoietic cells and immature enterocytes, are well recognised as being highly susceptible to the effects of radiation.

Early reactions following bone marrow transplants, such as mucositis, vomiting, diarrhoea, and anorexia, are well known in both adults and children. ${ }^{23}$ Delayed reactions, such as enteropathy ${ }^{4}$ and intrinsic or extrinsic intestinal obstruction following radiation injury to the gut have also been described. ${ }^{5}$ In a recent study we showed that zinc deficiency occurred in $67 \%$ of patients following bone marrow transplant, and was associated with adverse clinical sequelae related to sepsis. It was more common in younger patients and significantly linked with diarrhoea. ${ }^{6}$

Our understanding of the nature of gastrointestinal injury following bone marrow transplantation is at present fragmentary and mainly anecdotal. While mucosal damage, ${ }^{7}$ graftversus-host disease (GVHD), ${ }^{8}$ pancreatic damage, ${ }^{910}$ and microbial injury ${ }^{11}$ have all been reported after bone marrow transplants, the extent and the nature of any possible intestinal injury and its clinical and nutritional consequences have not been defined prospectively, either in adults or children. Management has tended to be entirely empirical. The aims of our study were therefore to define the natural history of the gastrointestinal injury associated with bone marrow transplantation, and to determine its effects on the clinical and biochemical indices of nutritional status and wellbeing.

\section{Methods}

SUBJECTS

Forty seven consecutive patients ( 28 males, 19 females; mean age 8.4 years) who underwent bone marrow transplantation over a period of two years were enrolled into the study after obtaining informed consent. The characteristics of the patients, their primary diagnoses and the type of graft received are given in table 1 .

\section{PROCEDURES}

Haematology, biochemistry, and gastrointestinal function

Serial haematological and biochemical analyses of blood urea, creatinine, electrolytes, calcium, phosphate, magnesium, albumin, alanine transaminase, aspartate transaminase, and alkaline phosphatase were carried out twice a week (more often if clinically indicated) during the period in hospital. Serial stool measurements ( $\alpha_{1}$-antitrypsin, chymotrypsin, steatocrit, reducing substances, and sugar chromatography) were carried out during periods of normal stools as well as during diarrhoeal episodes. In 14 asymptomatic patients it was possible to assess intestinal permeability during the conditioning regimen and after bone marrow transplantation, using mannitol/lactulose excretion ratios.

\section{Anthropometry}

Weight, height, and mid-arm circumference were measured weekly. 
Table 1 Clinical characteristics of patients ( $n=47)$

\begin{tabular}{ll}
\hline Mean (SD) age in years & $8.4(4.4)$ \\
Sex & 28 \\
Males & 19 \\
Females & 24 \\
Indications for BMT & 4 \\
Acute leukaemia & 4 \\
Chronic myelogenic anaemia & 3 \\
Solid tumours & 8 \\
Aplastic anaemia & 1 \\
$\beta$-Thalassaemia & 1 \\
Wiskott-Aldrich syndrome & 1 \\
Sickle cell anaemia & 1 \\
Primitive neurectodermal tumour & 18 \\
Myelodysplasia & 15 \\
Conditioning regimens & 10 \\
Cyclophosphamide and total body irradiation & 4 \\
Cyclophosphamide and busulphan $+/$ campath & \\
Cyclophosphamide, idarubicin, and total body irradiation & 42 \\
Melphalan & 5 \\
Graft & Allogeneic \\
Autologous & \\
\hline
\end{tabular}

BMT = bone marrow transplantation.

\section{Assessment of wellbeing}

Assessment of general wellbeing and activity was performed using the Lansky play performance scale weekly. ${ }^{12}$

\section{Conditioning regimens}

The regimens which were used for conditioning are given in table 1 . The indications for using each were as follows: cyclophosphamide and busulphan, with or without campath, were given primarily to children undergoing treatment for a haemoglobinopathy; a regimen using melphalan was given to children who had autografts for solid tumours; the use of cyclophosphamide in association with total body irradiation was the standard regimen for children with leukaemia; idarubicin was added in children who had certain high risk features, particularly specific chromosome abnormalities or very early relapse of acute leukaemia. In general the regimens lasted about eight days before bone marrow transplantation, exceptions being melphalan, which was given 48 hours before transplant, and the additional idarubicin, which was given on day 11 and 12 before transplant.

\section{Nutritional support}

Enteral nutrition (Nutrison Paediatric (Nutricia) for children weighing $<20 \mathrm{~kg}$ and Fortisip (Nutricia) for older children) was given to 21 patients; two with diarrhoea received Pepdite (SHS); parenteral nutrition (Hyperamine, Braun Medical) was given to 25 patients (19 with diarrhoea). Six children received enteral nutrition initially and parenteral nutrition afterwards, while one child received the opposite. Indications for providing nutritional support were: (1) malnutrition on admission (weight for height $\mathrm{z}$ score $<-1$ ); (2) weight loss of more than $5 \%$ and/or a decrease in mid-arm circumference of more than $10 \%$. Enteral nutrition was used in preference to parenteral nutrition when possible. Parenteral nutrition was offered when there was vomiting of the nasogastric tube ( $n=$ 6 ), unwillingness to have a nasogastric tube ( $n$ $=15)$, or diarrhoea $(n=4)$.

\section{CLINICAL DEFINITIONS}

\section{Diarrhoeal episodes}

The occurrence of diarrhoeal episodes (more than three loose stools per day for more than two consecutive days) was recorded. Each was investigated as follows: stool steatocrit, $\alpha_{1-}$ antitrypsin, stool chymotrypsin, stool electrolytes and osmolality, reducing substances, culture, clostridium toxins, and virology; where clinically indicated, a colonoscopy and/or an oesophago-gastroduodenoscopy was performed in order to rule out GVHD or cytomegalovirus (CMV) infection.

\section{Febrile episodes}

The occurrence of febrile episodes (temperature more than $38^{\circ} \mathrm{C}$ on at least one occasion per day for more than one day) was recorded. Each was investigated according to a previously published protocol. ${ }^{3}$

\section{Graft versus host disease}

GVHD was diagnosed clinically on the basis of a typical skin rash, abnormal liver function tests, and protracted diarrhoea, and was confirmed by biopsies of the affected organs (skin, liver, gut) where appropriate.

\section{Protein losing enteropathy}

Episodes of protein losing enteropathy were defined as a concentration of faecal $\alpha_{1}$ antitrypsin of $>2.2 \mathrm{mg} / \mathrm{g}$ dry stool. ${ }^{13}$

\section{Pancreatic insufficiency}

Pancreatic insufficiency was defined as a stool chymotrypsin activity of $<120 \mu \mathrm{g} / \mathrm{g}$ wet stool. ${ }^{14}$

\section{Steatorrhoea}

Steatorrhoea was defined as a stool steatocrit of $>2 \% .^{15}$

\section{Secretory diarrhoea}

Secretory diarrhoea was defined by a high concentration of faecal sodium ( $>60 \mathrm{mmol} / \mathrm{l}$ ) and an osmotic gap of less than $100 .^{16}$

\section{INTESTINAL PERMEABILITY}

Intestinal permeability was assessed by measuring urinary mannitol/lactulose ratios after oral dosing as previously described.$^{17}$ Increased intestinal permeability was defined as a mannitol/lactulose ratio of less than $3 .^{17}$

\section{STATISTICS}

Differences in stool $\alpha_{1}$-antitrypsin concentrations in children with diarrhoea due to GVHD or other causes, as well as mid-arm circumference $z$ scores in diarrhoeal/non-diarrhoeal patients, were assessed using the Student's $t$ test. The frequency of diarrhoea in children receiving idarubicin/cyclophosphamide was assessed using Fisher's exact test. All the remaining comparisons were carried out using the Mann-Whitney test.

\section{Results}

Thirty one diarrhoeal episodes (median duration 9.5 days) developed in 27 patients at a median of 10 days after bone marrow trans- 
plantation (range three days before to 49 days after transplantation). Idarubicin was associated with more frequent diarrhoea compared to cyclophosphamide plus total body irradiation ( $90 \% v 50 \%$ respectively, $\mathrm{p}=0.05)$, and of a longer duration: mean (SD) duration was 9.6 (8.7) v 3.9 (4.6) days respectively; $p=0.03$. The use of total body irradiation per se was not associated with more frequent or prolonged diarrhoea than other regimens.

Diarrhoea developed irrespective of the degree of wasting of the patients before transplantation or any other clinical features of gastrointestinal injury after transplantation (such as vomiting). However, diarrhoea was more often associated with the use of parenteral than enteral nutrition $(76 \%$ of the patients who received parenteral nutrition $v$ $19 \%$ of those who received enteral nutrition developed diarrhoea $(p=0.0003)$ ), and was of a relatively longer duration (mean (SD) duration 11.4 (11.7) $v 4.2$ (1.5) days respectively; $p=0.08$ ). Furthermore, in 15 patients who received parenteral nutrition diarrhoea developed after it had been started (mean time of onset, seven days after start of parenteral nutrition).

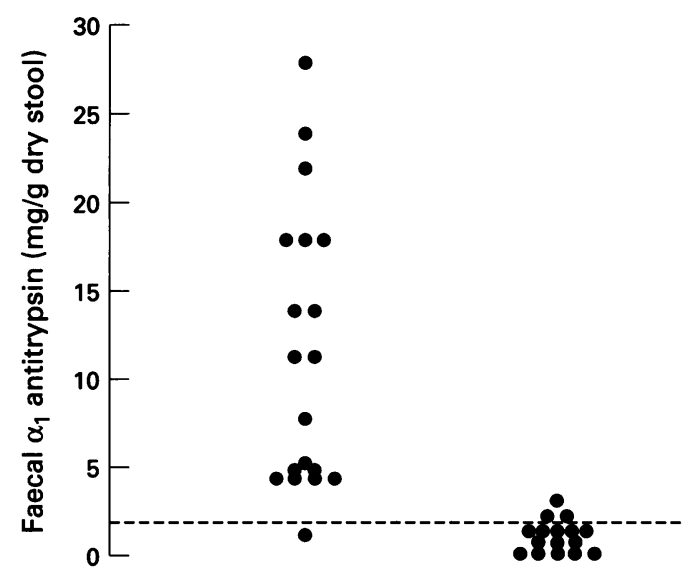

Diarrhoea Normal stool

Figure 1 Faecal $\alpha_{1}$-antitrypsin concentrations in diarrhoeal and normal stools after bone marrow transplant $(p<0.0001)$.

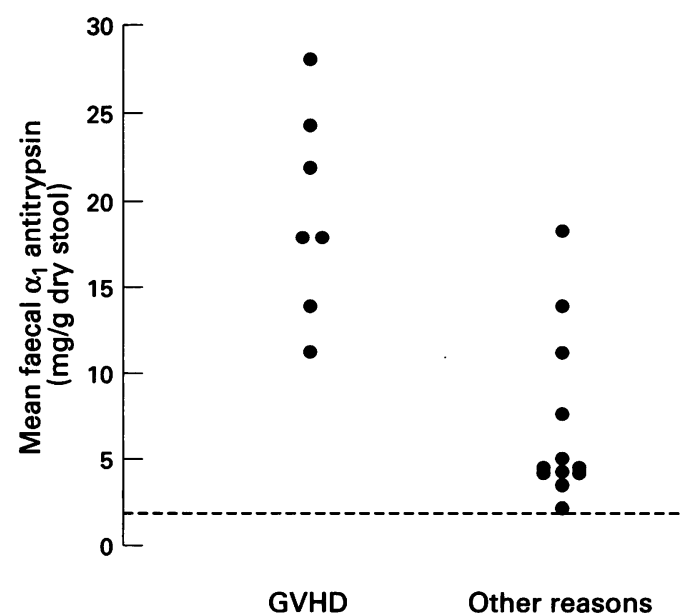

Figure 2 Faecal $\alpha_{1}$-antitrypsin concentrations in patients with and without graft versus host disease (GVHD) after bone marrow transplant $(p<0.0001)$.
A cause of diarrhoea was identified in 18 of the episodes as follows: gut GVHD, confirmed by rectal biopsy, in eight; skin and/or liver GVHD in six; suspected CMV colitis in one; rotavirus infection in three. Thirteen diarrhoeal episodes were of uncertain cause. However, six of them were associated with fever and neutropenia and were probably infectious, although no pathogenic micro-organisms were found in the stools; in two, $E$ coli and a faecal streptococcus were isolated from blood cultures. Twenty three of the diarrhoeal episodes and 53 of the normal stools in the same patients, as well as 23 of the normal stools in 10 of the children who did not develop diarrhoea, were assessed for protein losing enteropathy and pancreatic insufficiency. In the other eight diarrhoeal episodes, the tests failed, mainly because of difficulties in collecting the samples, particularly in young ill patients (very watery stools or contamination by urine).

Protein losing enteropathy was present in 21 (91\%) of the 23 investigated diarrhoeal episodes: mean (SD) stool $\alpha_{1}$-antitrypsin during diarrhoeal episodes was 10 (8.0) $\mathrm{mg} / \mathrm{g}$ dry stool, $v 1.1(0.8) \mathrm{mg} / \mathrm{g}$ dry stool in normal stools from the same patients $(p<0.0001)$ (fig 1). Protein losing enteropathy was more severe in GVHD than in other causes of diarrhoea (rotavirus enteritis, suspected CMV infection, uncertain cause): mean (SD) $\alpha_{1-}$ antitrypsin values during GVHD was 19.4 (5.8) $\mathrm{mg} / \mathrm{g}$ dry stool, $v 6.7$ (4.6) $\mathrm{mg} / \mathrm{g}$ dry stool with other causes of diarrhoea, $p<0.0001$ (fig 2). Furthermore, mild protein losing enteropathy was observed even in children without diarrhoea after bone marrow transplantation: mean (SD) $\alpha_{1}$-antitrypsin 3.6 (4.2) $\mathrm{mg} / \mathrm{g}$ dry stool.

A significant fall in serum albumin was observed following bone marrow transplant: mean (SD) serum albumin before transplant was $38(2.6) \mathrm{g} / \mathrm{l}, v 32$ (3.1) $\mathrm{g} / 1$ after transplant $(\mathrm{p}<0.0001)$. Hypoalbuminaemia was more severe in children with diarrhoea: mean (SD) minimum value of serum albumin in children with diarrhoea following bone marrow transplantation was $29(4.2) \mathrm{g} / \mathrm{l}, v 32(2.8) \mathrm{g} / \mathrm{l}$ in children without diarrhoea $(p=0.004)$. A significant negative correlation was found between the mean stool $\alpha_{1}$-antitrypsin concentrations and the minimum values of serum albumin during episodes of diarrhoea associated with protein losing enteropathy $(r=$ $-0.59 ; p=0.006$; fig 3 ), suggesting that protein losing enteropathy was functionally related to the induction of hypoalbuminaemia. This was supported further by the fact that even in children without diarrhoea a significant negative correlation was found between the stool $\alpha_{1}$-antitrypsin and serum albumin $(r=-0.47$; $\mathrm{p}=0.03$ ).

Impaired pancreatic exocrine function developed in 19 children. Eighteen of them had pancreatic insufficiency at a median of 20 days after bone marrow transplantation (range +2 to +133 ), while one child developed pancreatitis four months after transplantation and total body irradiation. Fourteen of the children showed pancreatic insufficiency (mean stool 
chymotrypsin $46 \mu \mathrm{g} / \mathrm{g}$ wet stool) during diarrhoeal episodes, but in four patients pancreatic insufficiency was a late complication, with the main clinical symptoms of absent weight gain and occasionally offensive stools. Steatorrhoea (mean stool steatocrit 14\%) was associated with only seven cases of pancreatic insufficiency $(40 \%)$. In children who developed pancreatic insufficiency during diarrhoeal episodes, serial measurements of stool chymotrypsin were performed after diarrhoea had resolved and showed that pancreatic insufficiency persisted for up to one month after the end of diarrhoea. In all four cases who developed late pancreatic insufficiency, bone marrow transplantation was associated with the use of total body irradiation.

Secretory diarrhoea was found in $\mathbf{1 0}$ of the 16 children (60\%) whose diarrhoeal stools were collected and were loose enough to test: mean (SD) faecal sodium concentration and osmotic gap were $97 \mathrm{mmol} / \mathrm{l}(23.4)$ and 32 (12.8) respectively. The causes of diarrhoea in this group were: GVHD in five, suspected CMV infection in one, rotavirus infection in one, and uncertain in three.

Positive reducing substances were found in 14 of the 23 investigated diarrhoeal episodes $(61 \%)$. The causes of diarrhoea were GVHD in seven, rotavirus infection in two, possible CMV infection in one, and uncertain four. Six diarrhoeal episodes had a secretory component as well as positive stool reducing sugars.

Diarrhoea had a profound negative effect on the nutritional status of the affected patients, despite the provision of parenteral nutrition in the majority: mean (SD) changes in weight for height $z$ scores in diarrhoeal versus nondiarrhoeal patients were $-0.54(0.6) v-0.12$ (0.4) respectively; $p=0.02$ (fig 4 ); and mean (SDI) changes in mid-arm circumference $z$ scores in diarrhoeal and non-diarrhoeal patients were $-0.33(0.4)$ and $+0.02(0.3)$ respectively; $p=0.008$.

Diarrhoea after bone marrow transplantation was associated with reduced wellbeing: median (range) of Lansky play performance score during diarrhoeal episodes and periods of normal stool in the same patients were 50

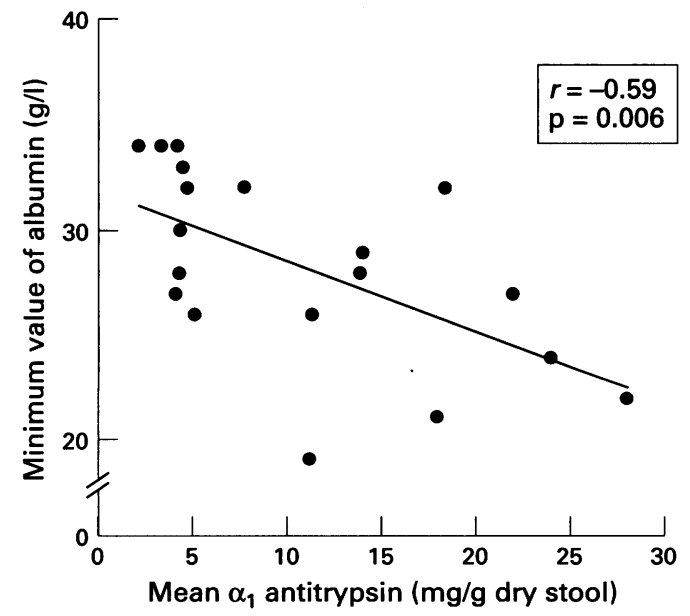

Figure 3 Correlation between stool $\alpha_{1}$-antitrypsin and serum albumin in children with diarrhoea after bone marrow transplant $(r=-0.59 ; p=0.006)$.
(10 to 80 ) compared with 70 (45 to 100 ) points, $p<0.0001$ (fig 5).

Intestinal permeability assessed by mannitol/ lactulose excretion ratios was found to be nor$\mathrm{mal}$ in 12 of the 14 investigated patients during the period of conditioning for bone marrow transplantation and in hospital following the transplant: median mannitol/lactulose corrected ratio 23.4 (normal $>3^{17}$ ).

\section{Discussion}

Our study showed that diarrhoea and hypoalbuminaemia were common following bone marrow transplants and were associated with adverse effects on nutritional status and wellbeing. Protein losing enteropathy and pancreatic insufficiency were also common after transplantation and were present in $91 \%$ and $78 \%$, respectively, of the diarrhoeal episodes investigated. Hypoalbuminaemia was worsened by diarrhoea and by protein losing enteropathy. Protein losing enteropathy complicating GVHD was more severe than when due to other causes. Intestinal permeability was normal in $86 \%$ of the investigated asymptomatic patients during conditioning therapies and after transplantation.
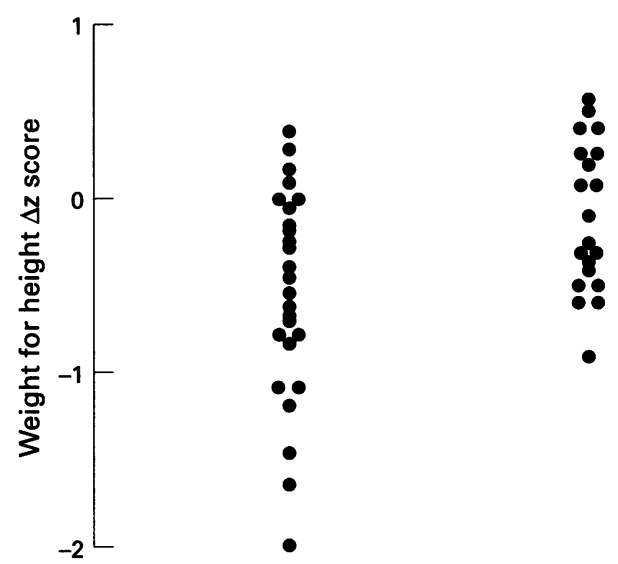

Diarrhoea

No diarrhoea

Figure 4 Maximum weight change in children with and without diarrhoea after bone marrow transplant $(p=$ 0.02).
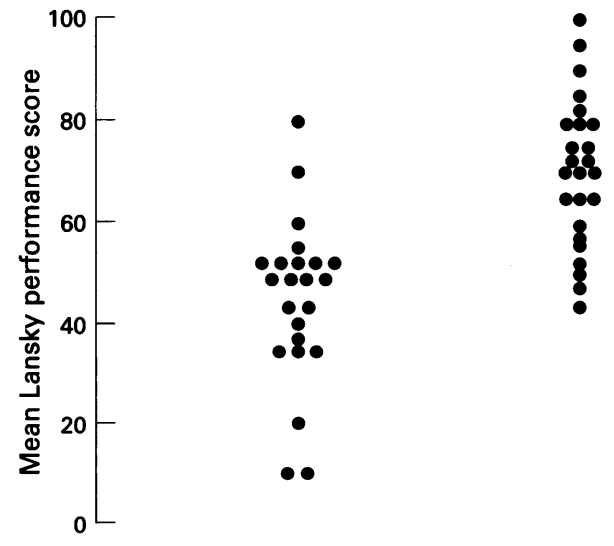

During diarrhoea

During normal stool

Figure 5 Lansky play performance after bone marrow transplant $(p<0.0001)$. 
This study has several limitations. First is the absence of a complete dataset of indices of gastrointestinal function. Despite the best efforts of highly motivated nursing colleagues, it was not always possible, or indeed acceptable, to obtain urine and stool samples for non-clinical purposes from severely ill children. Second was our inability to identify stool pathogens in several of the unexplained diarrhoeal episodes. Third is the limited use of a colonoscopy and biopsy (due to neutropenia and the increased risk of infection)-investigations which might have allowed us to determine the cause of intestinal damage in other cases. It must be stated, however, that all studies of diarrhoea in immunocompromised patients contain a substantial proportion of subjects in whom no stool pathogen is identified. ${ }^{18}$ Moreover, although the wider use of endoscopy in such patients has recently been advocated, its routine use in children with what is also self limiting diarrhoea is probably unjustified. ${ }^{19}$

Hypoalbuminaemia has previously been attributed to several factors, such as increased catabolism, decreased protein synthesis, fluid redistribution, protein loss from the gut due to GVHD,$^{20}$ and protein loss from pulmonary capillaries. $^{21}$ Although the aetiology of hypoalbuminaemia following bone marrow transplantation may be multifactorial, the significant negative correlation between the stool $\alpha_{1}$-antitrypsin and the fall in serum albumin suggested that protein loss from the gut was at least a major factor. Protein can be lost from the gut through an abnormal or inflamed mucosal surface. Radiation has been reported to have direct physical effects on tissues in animals, ${ }^{22}$ while GHVD has been shown to cause both a marked inflammation within the gut stroma and a degeneration and necrosis of the glandular epithelium and the crypt cells respectively. ${ }^{23}$ Protein losing enteropathy has also been reported in association with rotavirus infection in this context, ${ }^{24}$ while CMV infection was recently reported to simulate GVHD histologically. ${ }^{25}$

This study showed that carbohydrate intolerance was common during diarrhoea. Radiation has been reported to cause varying degrees of villous atrophy ${ }^{4}$ associated with functional changes such as disaccharide and fat malabsorption. ${ }^{26}$ Furthermore, an acceleration of orocaecal transit, associated with lactose and bile salt malabsorption, was recently reported in adults after abdominal irradiation. ${ }^{27}$

In line with recent anecdotal reports, ${ }^{9} 10$ our study showed that pancreatic exocrine function may be impaired by bone marrow transplantation. Indeed, the incidence of transient pancreatic insufficiency following transplantation in our study was high. However, fat malabsorption was not common, probably because of anorexia and low oral fat intake during diarrhoea, or alternatively because lipase secretion may have not fallen to the very low level required for fat malabsorption to occur.

The pathogenesis of pancreatic insufficiency following bone marrow transplantation has not been studied. GVHD has been implicated in the induction of pancreatic insufficiency in humans, in whom necropsy features of GVHD were reported in the pancreas. ${ }^{28}$ Similar features have been found in experimental models of GVHD. ${ }^{29}$ Total body irradiation and chemotherapy have been reported to induce pancreatitis following bone marrow transplantation. $^{10}$ Interestingly, one of our patients developed pancreatitis three months after bone marrow transplant and total body irradiation.

In contrast to previous reports in adults suggesting that the conditioning regimen leads to small bowel mucosal damage, ${ }^{30}{ }^{31}$ we found that intestinal permeability was normal in children without diarrhoea during the conditioning regimen and for up to six weeks after conditioning. Parrilli et al ${ }^{30}$ reported a more than doubled intestinal permeability as early as the second day of treatment in all of nine patients undergoing cytotoxic chemotherapy. Fegan et $a l^{31}$ reported an increased intestinal permeability peaking one to two weeks after conditioning in adults undergoing bone marrow transplantation. Intestinal permeability in that study was significantly increased in patients older than 30 years, was unrelated to the use of total body irradiation, and was correlated with the severity of gastrointestinal disturbance. We were unable to assess intestinal permeability during diarrhoeal episodes for three main reasons: unwillingness of some of the patients to drink the solution while feeling poorly; vomiting of the solution; or to the contamination of urine collection by diarrhoea especially in younger and female patients.

The gastrointestinal disturbance following bone marrow transplantation was shown to be associated with a marked adverse effect on nutritional status and on the wellbeing of affected patients, despite the provision of total parenteral nutrition to most of them. The nutritional management of the gastrointestinal sequelae of bone marrow transplantation is therefore challenging. The fact that $50 \%$ of diarrhoeal episodes developed after the start of parenteral nutrition poses the question of whether enteral feeding might have been more protective to the gut and more beneficial to the patients. Parenteral nutrition is associated with atrophic changes in the gut and reduced pancreatic function in animals. ${ }^{32}$ It is also associated with a higher infection rate in critically ill patients than enteral nutrition. ${ }^{34}$ Bacterial translocation to the circulation from the gut has been implicated as a contributory factor. ${ }^{35}$ Enteral nutrition has been shown to preserve intestinal barrier function in animal models, ${ }^{36}$ and to improve immunity, reduce infection rate, and improve outcome in humans. ${ }^{37}$ Recent studies have shown a beneficial effect of dietary supplements such as glutamine and fermentable fibre on gut mucosal turnover, thereby protecting intestinal barrier function from a variety of injurious agents. ${ }^{38} 39$

We conclude that gastrointestinal injury following bone marrow transplantation is complex and has profound effects on nutritional status and wellbeing of the patients. Further prospective studies evaluating the preferred route of nutrient administration are required. 
We wish to thank the nursing staff of the BMT unit for their enormous contribution to the study; Ms Karen Rose, the play specialist of the BMT unit for undertaking the weekly play perormance assessments; Steve Johnson, the laboratory manage e Child Health for his contribution to the laboatory work; and Dr K Nathavitharana, consultant paediatrician, for his valuable comments during the design of the study. The study was sponsored by local Bone Marrow Transplan Research Special Funds.

1 Armitage JO. Bone marrow transplantation. $N$ Engl Med 994:330:827-38.

2 Wolford JL, McDonald GB. A problem-oriented aproach to intestinal and liver disease after marrow transplantation. $f$ Clin Gastroenterol 1988;10:419-33.

3 Papadopoulou A, Nathavitharana KA, Williams MD, Darbyshire PJ, Booth IW. Diarrhoea and weight loss after bone marrow transplantation. Pediatr Hematol Oncol 1994; 11:601-11.

4 Donaldson SS, Jundt S, Ricour C, Sarrazin D, Lemerle J, Schweisguth O. Radiation enteritis in children. Cancer 1975;35:1167-78.

5 Potish R, Thomas KJ, Levitt S. Factors predisposing to radiation-related small bowel damage. Radiology 1979;132: 479-82.

6 Papadopoulou A, Nathavitharana KA, Williams MD, Darbyshire PJ, Booth IW. Diagnosis and clinical associations of zinc depletion following bone marrow transplantation. Arch Dis Child 1995;74:328-31.

7 McDonald GB, Shulman HM, Sullivan KM, Spencer GD. Intestinal and hepatic complications of human bone marrow transplantation: part I. Gastroenterology 1986;90: 460-77.

8 Ferrara JLM, Deeg HJ. Graft versus host disease. $N$ Engl $\mathcal{Y}$ Med 1991;324:667-74

9 Jurges E, O'Donohoe J. Pancreatic insufficiency after bone marrow transplantation. Lancet 1991;338:517.

10 Werlin SL, Casper J, Antonson D, Calabro C. Pancreatitis associated with bone marrow transplantation in children. Bone Marrow Transplant 1992;10:65-9.

11 Blakey JL, Barnes GL, Bishop RF, Ekert H. Infectious diarrhoea in children undergoing bone-marrow transplantation. Aust NZ $¥$ Med 1989;9:31-6.

12 Lansky SB, List MA, Lansky LL, Ritter-Sterr C, Miller DR. The measurement of performance in childhood cancer The measurement of performanc

13 Magazzu G, Iacono G, di Pasquale G, et al. Reliability and usefulness of random fecal $\alpha_{1}$-antitrypsin concentration: further simplification of the method. $\mathcal{F}$ Paediatr Gastroenterol Nutr 1985;3:402-7.

14 Brown GA, Halliday RB, Turner PJ, Smalley CA. Faecal chymotrypsin concentrations in neonates with cystic fibrosis and healthy controls. Arch Dis Child 1988;63:1229-33.

15 Iacono G, Carroccio A, Cavatgio F, et al. Steatocrit test: normal range and physiological variations in infants. $₹$ Paediatr Gastroenterol Nutr 1990;11:53-7.

16 Ladefogen $\mathrm{K}$, Schaffalitzky de Muckadell OB, Jarnum S. Faecal osmolality and electrolyte concentrations in chronic diarrhoea: do they provide diagnostic clues? Scand $\mathfrak{f}$ Gastroenterol 1987;22:813-20.

17 Nathavitharana KA, Lloyd DR, Raafat F, Brown GA McNeish AS. Urinary mannitol-lactulose excretion ratios and jejunal

18 Crotty $\mathrm{P}$, Smallwood RA Investigating diarrhoea in patients with acquired immunodeficiency syndrome. Gastroenterology 1996;110:296-310.
19 Wilcox CM, Schwartz BA, Cotsonis G, Thompson SE. Chronic unexplained diarrhoea in human immunodeficiency virus infection: determination of the best diagnostic approach. Gastroenterology 1996;110:30-7

20 Weisdorf SA, Salati JA, Longsdorf NKC, Sharp HL. Graftversus-host disease of the intestine: a protein losing enteropathy characterised by faecal $\alpha_{1}$-antitrypsin. Gastroenterology 1983;85:1076-81.

21 Guiot HFL, Jemond J, Klasen E, et al. Protein loss during acute graft versus host disease: diagnostic and clinical significance. Eur 7 Haematol 1987;38:187-96.

22 Buel MG, Harding RK. Proinflammatory effects of local abdominal irradiation on rat gastrointestinal tract. Dig Dis Sci 1989;34:390-9.

23 Appleton AL, Sviland L, Pearson ADJ, Green MA, Eastham EJ, Malcolm AJ. The need for endoscopic biopsy in the diagnosis of upper gastrointestinal oraft-versus-host disease. $₹$ Pediatr Gastroenterol Nutr 1993;16:183-5.

24 Willoughby RE, We SB, Yolken RH. Non-group A rotavirus infection associated with severe gastroenteritis in a bone infection associated with severe gastroenteritis in a bone

25 Eirrow transplant patient. Pediatr Infect Dis 1988;7:133-5. CMV infection and acute intestinal GVHD in marrow transplant recipients with severe diarrheoa. Bone Marrow Transplant 1994;14:955-63.

26 Beer WH, Fan A, Halsted CH. Clinical and nutritional implications of radiation enteritis. Am $\mathcal{F}$ Clin Nutr 1985;41: 85-91.

27 Fernandez-Banares F, Villa S, Esteve M, et al. Acute effects of abdominopelvic irradiation on the orocecal transit time: its relation to clinical symptoms, and bile salt and lactose malabsorptio. Am f Gastroenterol 1991;86:1771-7.

28 Nakamura NA. Clinopathological study of 15 autopsy cases with allogenic bone marrow transplantation in special with allogenic bone marrow transplantation in special 39:641-4.

29 Thomas A, Seemayer JG, Bartner EC, Stapp W. Acute graft versus host reaction in the pancreas. Transplantation 1983; 35:72-7.

30 Parrilli G, Laffaioli RV, Capuano G, Budillon G, Bianco AR. Changes in intestinal permeability to lactulose induced by cytotoxic chemotherapy. Cancer Treat Rep 1982;66:1435-6.

31 Fegan $\mathrm{C}$, Poynton $\mathrm{CH}$, Whittaker JA. The gut mucosal barrier in bone marrow transplantation. Bone Marrow Transplant 1990;5:373-7.

32 Hughes CA, Dowling RH. Speed of onset of adaptive mucosal hypoplasia and hypofunction in the intestine of parenterally fed rats. Clin Sci 1980;59:317-27.

33 Hughes CA, Prince A, Dowling RH. Speed of change in pancreatic masses and in intestinal bacteriology in pancreatic masses and in intestinal bac

34 Kudsk KA. Gut mucosal nutritional support-enteral nutrition as primary therapy after multiple system trauma. Gut 994;suppl 1:S52-4.

35 Alexander JW. Nutrition and translocation. fournal of Parenteral and Enteral Nutrition 1990;14(suppl):170-4S.

36 Inoue S, Epstein MD, Alexander JW, et al. Prevention of yeast translocation across the gut by a single enteral feeding after burn injury. Fournal of Parenteral and Enteral Nutrition 1989;13:565-71.

37 Moore FA, Moore EE, Jones TN, McCroskey BL, Peterson VM. TEN versus TPN following major torso trauma: reduced septic morbidity. $\mathcal{F}$ Trauma 1989;9:916-23.

38 Deitch EA. Bacterial translocation: the influence of dietary variables. Gut 1994;suppl 1:S23-7.

39 Souba WW, Klimberg VS, Hautamaki RD, et al. Oral glutamine reduces bacterial translocation following abdominal radiation. $\mathcal{F}$ Surg Res 1990;48:1-5. 